\title{
WITT'S THEOREM AND THE ISOMETRY OF LATTICES
}

\author{
O. T. O'MEARA
}

1. Introduction. The cancellation law for quadratic forms as originally proved by Witt [10] states that if $\mathcal{F}+G$ and $\mathcal{F}+\mathcal{H C}$ are field equivalent then so are $G$ and $\mathfrak{F C}$. In general this result is not valid under ring equivalence but some work has been done in this direction, notably by Jones and Durfee in the local theory and by Eichler and Kneser in the ring of rational integers. Although the integral formulation of Witt's theorem holds over any local field in which 2 is a unit $[2 ; 5]$, it is not true over the 2-adic integers [2]; but in that case it is known [6] that $G \cong \mathscr{F}$ provided that $\mathcal{F}$ is of unit determinant, $G=2 G^{\prime}$ and $\mathfrak{H C}=2 \mathfrak{H C}^{\prime}$. The author [9] has extended this result to any local field in which 2 is a prime; also to the ramified case, but with the restriction that $\mathcal{F}$ be a form of one variable only. Our first aim in this paper is to remove this restriction. Secondly, to prove that $G$ and $\mathcal{F C}$ are equivalent provided that $\mathcal{F}$ is the orthogonal sum of totally isotropic binary forms, that is forms of the type

$$
\left(\begin{array}{cc}
0 & \pi^{\lambda(i)} \\
\pi^{\lambda(i)} & 0
\end{array}\right),
$$

where the $\lambda(i)$ are arbitrary integers.

2. Notation. The scalars will be taken from a field $F$ of characteristic unequal to 2, provided with a non-Archimedean, complete and discrete valuation [1] and having a perfect residue class field; 0 will be the ring of integers and $\pi$ a prime element in $F$; since the cancellation law has been shown to hold for any local field in which 2 is a unit $[2 ; 5]$, we shall assume throughout that $|2|<1$ holds in $F$; the letter $e$ will be used to denote the ordinal of 2, i.e. $\left|\pi^{e}\right|=|2|$; then our assumption can be expressed by the relation $e=$ ord $2 \geqq 1$. A specific element $a \pi^{t}$ will sometimes be written as $\left[\pi^{t}\right]$ if $|a|=1$ and as $\left\{\pi^{t}\right\}$ if $a \in 0$; thus $\alpha=\left[\pi^{t}\right], 1 \beta=\left[\pi^{t}\right], \gamma=\left\{\pi^{t}\right\}$ implies that $|\alpha|$ $=|\beta| \geqq|\gamma|$.

All proofs will be geometrical. The notation will be similar to that in [9]. If $V$ is an $n$-dimensional $F$-space with scalar product $X \cdot Y$

Received by the editors January 17, 1955. 
and if $L$ is a lattice $[3 ; 9]$ in $V$, we say that $\xi_{1}, \cdots, \xi_{n}$ is a (minimal) basis for $L$ if $L=0 \xi_{1}+\cdots+0 \xi_{n}$. If $\mathcal{L}$ is the quadratic form associated with this basis and if $\Lambda$ is the corresponding matrix, we write $\mathcal{L} \cong \Lambda \cong L$. If $K$ is another lattice in $V$ and if there exists an isometry carrying $L$ onto $K$, we write $L \cong K, \Lambda \cong \mathrm{K}$ and $\mathcal{L} \cong K$. The determinant of $L$ is defined to be the determinant of the matrix $\Lambda$; we assume that $\operatorname{det} L \neq 0$.

The ideal generated by $X^{2}, X \in L$, is called the norm of $L$ and will be written $N(L)$; if $N(L) \subseteq 0$ we say that $L$ is integral. If $X \in L$, we define $\mathfrak{a}_{L}(X)$, or simply $\mathfrak{a}(X)$, as the ideal generated by $X \cdot Z$ as $Z$ runs through $L$; the ideal generated by $\mathfrak{a}(X), X \in L$, will be written $\mathfrak{a}(L)$; if $\mathfrak{a}(L) \subseteq \mathrm{o}$ we say that $L$ is totally integral.

If $L$ is a totally integral lattice of unit determinant it has a canonical basis, i.e. a basis $\left(\xi_{\lambda}\right)$ such that (i) if $N(L)=0$, then $\xi_{\lambda} \xi_{\mu}=0$ for all $\lambda \neq \mu$; (ii) if $N(L) \subseteq(\pi) \mathrm{o}$, then $n$ is even and $\xi_{2 r-1} \xi_{2 r}=1, \xi_{2 r-1} \xi_{\lambda}=0$ $=\xi_{2 r} \xi_{\lambda}$ for $\lambda \neq 2 r-1,2 r$ and for all $r, 1 \leqq r \leqq n / 2$. In the first case we say that $L$ is proper; in the second, improper. If $L$ is arbitrary, it has an orthogonal decomposition

$$
L=L_{1}\left(\pi^{s(1)}\right) \oplus \cdots \oplus L_{r}\left(\pi^{s(r)}\right)
$$

where $s(1)<s(2)<\cdots<s(r)$ and the forms $\pi^{-s(\lambda)} \mathcal{L}_{\lambda}\left(\pi^{s(\lambda)}\right)$ are totally integral, of unit determinant. The basis derived from the canonical bases of the $L_{\lambda}\left(\pi^{8(\lambda)}\right)$ in the obvious way, is called a canonical basis for $L$. If $L$ has another decomposition

$$
L=K_{1}\left(\pi^{t(1)}\right) \oplus \cdots \oplus K_{q}\left(\pi^{t(q)}\right),
$$

then

$$
\begin{gathered}
s(\lambda)=t(\lambda), \quad \operatorname{dim} L_{\lambda}=\operatorname{dim} K_{\lambda}, \\
L_{\lambda} \text { proper } \Leftrightarrow K_{\lambda} \text { proper. }
\end{gathered}
$$

Let $\left(\xi_{\lambda}\right)$ be canonical; we say that $\xi_{i}$ is a proper basis element if $\xi_{i} \xi_{\lambda}=0$ for all $\lambda \neq i$; otherwise it is improper. Note that $a\left(\xi_{i}\right)=\left(\xi_{i}^{2}\right)_{0}$ if and only if $\xi_{i}$ is proper.

The proof that $L$ has a canonical basis and of the equations (2.2) can be found in [9]; it is rather well known for the rational 2-adic integers [7]. If two lattices (not necessarily isometric) have the same invariants (2.2) and (2.2a) we say that they are of the same type.

Some of our proofs will rely on the following "one-dimensional" result, already proved in [9, Theorem 5.1]:

THEOREM 2.1. Let the lattice $L$ have two decompositions $0 \xi \oplus L_{1}=L$ $=\mathfrak{o} \oplus \oplus K_{1}$ with $\xi^{2}=\epsilon=\eta^{2}$ and $|\epsilon|=1$. If $\mathfrak{a}\left(L_{1}\right) \subseteq(2) \mathfrak{o}$, then $L_{1} \cong K_{1}$. 
3. The derived canonical basis. Let $\left(\xi_{\lambda}\right)$ be a canonical basis for $L$. Starting with two fixed, distinct and orthogonal basis elements $\xi_{i}, \xi_{j}$ and with a given integer $\alpha$, we shall derive a new canonical basis $\left(\boldsymbol{\Xi}_{\lambda}\right)$ from the old one $\left(\xi_{\lambda}\right)$. The operation of passing from the one basis to the other will be denoted by the symbol

$$
\text { op }\left(\xi_{i} \rightarrow \xi_{i}+\alpha \xi_{j}\right) \text {; }
$$

the value of $\Xi_{i}$ will be given by the equation $\Xi_{i}=\xi_{i}+\alpha \xi_{j}$; thus

$$
\Xi_{i}^{2}=\xi_{i}^{2}+\alpha^{2} \xi_{j}^{2}
$$

we define $\Xi_{\lambda}=\xi_{\lambda}$ at those values of $\lambda$ for which $\lambda \neq i, j$ and $\xi_{\lambda} \xi_{j}=0$. Most of the $\Xi_{\lambda}$ are now settled; to complete the definition of the basis $\left(\Xi_{\lambda}\right)$ we must specify the vector $\Xi_{j}$ and (when $\xi_{j}$ is improper with $\xi_{j} \xi_{j+1} \neq 0$, say) the vector $\Xi_{j+1}$. To ensure that the constructions involved in defining the remaining $\boldsymbol{\Xi}_{\lambda}$ yield a canonical basis, we must assume that

$$
\alpha \in \mathfrak{D}, \quad \alpha \mathfrak{a}\left(\xi_{j}\right) / \mathfrak{a}\left(\xi_{i}\right) \subseteq \mathfrak{D},
$$

(3.3) $\left|\Xi_{i}^{2}\right|=\left|\mathfrak{a}\left(\xi_{i}\right)\right|$ if $\xi_{i}$ proper, $\left|\Xi_{i}^{2}\right|<\left|\mathfrak{a}\left(\xi_{i}\right)\right|$ if $\xi_{i}$ improper.

CASE 1. $\xi_{i}, \xi_{j}$ proper. Then $\alpha \xi_{j}^{2} / \Xi_{i}^{2} \in \mathcal{0}$ by (3.2) and (3.3); define $\Xi_{j}=\xi_{j}-\left(\alpha \xi_{j}^{2} / \Xi_{i}^{2}\right) \Xi_{i}$; then $\left(\Xi_{\lambda}\right)$ is canonical and

$$
\Xi_{j}^{2}=\xi_{j}^{2}-\alpha^{2}\left(\xi_{j}^{2}\right)^{2} / \Xi_{i}^{2} \text {. }
$$

CASE 2. $\xi_{i}$ improper, $\xi_{j}$ proper. Let $\xi_{i \pm 1}=\Xi_{i \pm 1}$ be the basis vector that is not orthogonal to $\xi_{i}$. Put

$$
\begin{aligned}
& A=-\alpha \xi_{i}^{2} \Xi_{i \pm 1}^{2} /\left(\Xi_{i}^{2} \Xi_{i \pm 1}^{2}-\left(\Xi_{i} \Xi_{i \pm 1}\right)^{2}\right) \in \mathrm{o}, \\
& B=\alpha \xi_{j}^{2}\left(\Xi_{i} \Xi_{i \pm 1}\right) /\left(\Xi_{i}^{2} \Xi_{i \pm 1}^{2}-\left(\Xi_{i} \Xi_{i \pm 1}\right)^{2}\right) \in \mathrm{o}
\end{aligned}
$$

and define $\boldsymbol{\Xi}_{j}=\xi_{j}+A \boldsymbol{\Xi}_{i}+B \boldsymbol{\Xi}_{i \pm 1}$; then $\boldsymbol{\Xi}_{j} \boldsymbol{\Xi}_{i}=0=\boldsymbol{\Xi}_{j} \boldsymbol{\Xi}_{i \pm 1}$ by choice of $A$ and $B$; and $\left(\Xi_{\lambda}\right)$ is canonical in virtue of (2.2) and (2.2a). Direct computation shows that

$$
\Xi_{j}^{2}=\xi_{i}^{2}+\left[\alpha^{2}\left(\xi_{j}^{2}\right)^{2} \Xi_{i \pm 1}^{2} /\left(\Xi_{i} \Xi_{i \pm 1}\right)^{2}\right]
$$

CASE 3. $\xi_{i}$ proper, $\xi_{j} \xi_{j+1} \neq 0$. Define $\Xi_{j}=\xi_{j}-\left(\alpha \xi_{j}^{2} / \Xi_{i}^{2}\right) \Xi_{i}$ and $\Xi_{j+1}^{\prime}$ $=\xi_{j+1}-\left(\alpha \xi_{j} \xi_{j+1} / \Xi_{l}^{2}\right) \Xi_{i} ;$ then $\Xi_{j} \Xi_{j+1}^{\prime}=\xi_{j} \xi_{j+1}\left(1-\alpha^{2} \xi_{j}^{2} / \Xi_{l}^{2}\right) ;$ put $\Xi_{j+1}$ $=\boldsymbol{\Xi}_{j+1}^{\prime}\left(\xi_{j} \xi_{j+1} / \boldsymbol{\Xi}_{j} \boldsymbol{\Xi}_{j+1}^{\prime}\right)$. Then $\left(\boldsymbol{\Xi}_{\lambda}\right)$ is canonical and direct computation yields $\boldsymbol{\Xi}_{j} \boldsymbol{\Xi}_{j+1}=\xi_{j} \xi_{j+1}$ and

$$
\Xi_{j}^{2}=\xi_{j}^{2}-\alpha^{2}\left(\xi_{j}^{2}\right)^{2} / \Xi_{i}^{2}, \quad \Xi_{j+1}^{2}=\xi_{j+1}^{2}+\left[\alpha^{2}\left(\xi_{j} \xi_{j+1}\right)^{2} / \Xi_{i}^{2}\right] .
$$


CASE 4. $\xi_{i}$ improper, $\xi_{j} \xi_{j+1} \neq 0$. Define $\Xi_{j}$ as in Case 2 and put $\Xi_{j+1}^{\prime}=\xi_{j+1}+A^{\prime} \boldsymbol{\Xi}_{i}+B^{\prime} \Xi_{i \pm 1}$ where

$$
\begin{aligned}
& A^{\prime}=-\alpha\left(\xi_{j} \xi_{j+1}\right) \Xi_{i \pm 1}^{2} /\left(\Xi_{i}^{2} \Xi_{i \pm 1}^{2}-\left(\Xi_{i} \Xi_{i \pm 1}\right)^{2}\right) \in \mathrm{o}, \\
& B^{\prime}=\alpha\left(\xi_{j} \xi_{j+1}\right)\left(\Xi_{i} \Xi_{i \pm 1}\right) /\left(\Xi_{i}^{2} \Xi_{i \pm 1}^{2}-\left(\Xi_{i} \Xi_{i \pm 1}\right)^{2}\right) \in \mathrm{o} .
\end{aligned}
$$

The fact that $A^{\prime}$ and $B^{\prime}$ are integers follows from (3.2) and (3.3). Then $\boldsymbol{\Xi}_{j}$ and $\boldsymbol{\Xi}_{j+1}^{\prime}$ are orthogonal to $\mathrm{D} \boldsymbol{\Xi}_{i}+\mathrm{D} \boldsymbol{\Xi}_{i \pm 1}$. Also $\boldsymbol{\Xi}_{j+1}^{\prime} \boldsymbol{\Xi}_{j}=\xi_{j+1} \xi_{j}$ $\cdot\left(1+\left\{\alpha^{2} \xi_{j}^{2} \Xi_{i \pm 1}^{2} /\left(\Xi_{i} \Xi_{i \pm 1}\right)^{2}\right\}\right)$. Define $\boldsymbol{\Xi}_{j+1}=\Xi_{j+1}^{\prime}\left(\xi_{j+1} \xi_{j} / \Xi_{j+1}^{\prime} \Xi_{j}\right)$. Then $\left(\boldsymbol{\Xi}_{\lambda}\right)$ is canonical and direct computation shows that $\Xi_{j} \Xi_{j+1}=\xi_{j} \xi_{j+1}$ and

$$
\begin{aligned}
\Xi_{j}^{2} & =\xi_{j}^{2}+\left[\alpha^{2}\left(\xi_{j}^{2}\right)^{2} \Xi_{i \pm 1}^{2} /\left(\Xi_{i} \Xi_{i \pm 1}\right)^{2}\right], \\
\Xi_{j+1}^{2} & =\xi_{j+1}^{2}+\left[\alpha^{2}\left(\xi_{j} \xi_{j+1}\right)^{2} \Xi_{i \pm 1}^{2} /\left(\Xi_{i} \Xi_{i \pm 1}\right)^{2}\right] .
\end{aligned}
$$

This completes the definition of the basis $\left(\Xi_{\lambda}\right)$.

In the definition of op $\left(\xi_{i} \rightarrow \xi_{i}+\alpha \xi_{j}\right)$ we have assumed that $i \neq j$ and that $\xi_{i} \xi_{j}=0$. We now define op $\left(\xi_{i} \rightarrow \xi_{i}+\alpha \xi_{i}\right)$ in a similar manner:

CASE 5. $i=j,|1+\alpha|=1$. Define $\Xi_{i}=\xi_{i}+\alpha \xi_{i}$; if $\xi_{i \pm 1} \xi_{i} \neq 0$, put $\Xi_{i \pm 1}=(1+\alpha)^{-1} \xi_{i \pm 1}$; set the remaining $\Xi_{\lambda}=\xi_{\lambda}$. Note that

$$
|\alpha|>|2| \Rightarrow \Xi_{i}^{2}=\xi_{i}^{2}+\alpha^{2} \xi_{i}^{2}+\left\{\pi \alpha^{2} \xi_{i}^{2}\right\} \text {. }
$$

Finally we define the symbol op $\left(\xi_{i+1} \rightarrow \xi_{i+1}+\alpha \xi_{i}\right)$ when $\xi_{i} \xi_{i+1} \neq 0$. Note that in this case neither $\boldsymbol{\Xi}_{i}$ nor $\boldsymbol{\Xi}_{i+1}$ will be defined in the usual way.

CASE 6. $\xi_{i} \xi_{i+1} \neq 0, \alpha \in$ o. Define $\Xi_{i}=\xi_{i}$ and $\Xi_{i+1}^{\prime}=\xi_{i+1}+\alpha \xi_{i}$; we then have $\Xi_{i} \Xi_{i+1}^{\prime}=\xi_{i} \xi_{i+1}\left(1+\alpha \xi_{i}^{2} / \xi_{i} \xi_{i+1}\right)$ and we put

$$
\Xi_{i+1}=\Xi_{i+1}^{\prime}\left(\xi_{i} \xi_{i+1} / \Xi_{i} \Xi_{i+1}^{\prime}\right)
$$

Set the remaining $\Xi_{\lambda}=\xi_{\lambda}$. Note that

$$
\begin{aligned}
& |\alpha|>\left|2 \xi_{i} \xi_{i+1} / \xi_{i}^{2}\right| \Rightarrow \Xi_{i+1}^{2}=\xi_{i+1}^{2}+\alpha^{2} \xi_{i}^{2}+\left\{\pi \alpha^{2} \xi_{i}^{2}\right\}, \\
& |\alpha|<\left|2 \xi_{i} \xi_{i+1} / \xi_{i}^{2}\right| \Rightarrow \Xi_{i+1}^{2}=\xi_{i+1}^{2}+2 \alpha \xi_{i} \xi_{i+1}+\left\{2 \pi \alpha \xi_{i} \xi_{i+1}\right\} .
\end{aligned}
$$

We conclude this section with some lemmas.

LEMMA 3.1. If $\xi^{2}=a+\{4 \pi\}$ where $a$ is a unit, then $\mathrm{o} \xi \cong a$.

Proof. Apply Hensel's lemma [1].

The next lemma is a necessary modification of a result of the author [9] and a slight sharpening of the binary formulation of Theorem 2 in [2]. Its corollary is weaker than Theorem 14.3 in [9] but will meet the requirements of this paper. 
LEMMA 3.2. Let $L=0 \xi_{1}+\mathrm{o} \xi_{2}$ be an improper lattice of norm $\left(\pi^{\nu}\right) \mathrm{o}$ such that $\xi_{1} \xi_{2}=1$ and $\xi_{2}^{2}=a+\left[\pi^{\Delta}\right]$. Suppose that either (1) $\left|\xi_{1}^{2}\right|=\left|\pi^{\prime}\right|$ and $\Delta \geqq 2 e-\nu+1$; or (2) $\Delta \geqq 2 e+1$; then there is an $X$ in $L$ such that $L=\mathrm{o} \xi_{1}+\mathrm{o} X, \xi_{1} X=1$ and $X^{2}=a$.

Proof. The quadratic equation

$$
\alpha^{2}\left(\xi_{1}^{2}-a\left(\xi_{1}^{2}\right)^{2}\right)+2 \alpha\left(1-a \xi_{1}^{2}\right)+\left(\xi_{2}^{2}-a\right)=0
$$

can be written in the form $\alpha^{2}+\left[2 \pi^{-\nu}\right] \alpha+\left\{\pi^{26-2 \nu+1}\right\}=0$ which has an integral solution $\alpha_{0}$ by Hensel's lemma [1]. Then

$$
X=\left(\xi_{2}+\alpha_{0} \xi_{1}\right) /\left(1+\alpha_{0} \xi_{1}^{2}\right)
$$

is the vector required.

The second part of the lemma is proved in the same way.

CoROllaRy 3.2. Let $L=\mathrm{o} \xi_{1}+\mathrm{o} \xi_{2}$ and $K=\mathrm{o} \eta_{1}+\mathrm{o} \eta_{2}$ be improper lattices of the same norm $\left(\pi^{\nu}\right) \mathrm{D}$ and of the same determinant, such that $\xi_{1}^{2}=\eta_{1}^{2}=\left[\pi^{\nu}\right], \xi_{1} \xi_{2}=1=\eta_{1} \eta_{2}$ and $\xi_{2}^{2}=\eta_{2}^{2}+\left\{\pi^{2 e-\nu}\right\}$. Then $L \cong K$.

Proof. Since $L$ and $K$ are evidently field equivalent we can assume that they are in the same metric space $V$. Define $f=e$ or $f=e+1$ in such a way that $f+\nu$ is even and put $L^{\prime}=0 \xi_{0} \oplus L, K^{\prime}=0 \xi_{0} \oplus K$, where $\xi_{0}^{2}=\pi^{-f}$. Applying op $\left(\eta_{2} \rightarrow \eta_{2}+\alpha \xi_{0}\right)$ with a suitable $\alpha$ for which $\eta_{2}^{2}+\alpha^{2} \xi_{0}^{2} \equiv \xi_{2}^{2}\left(\bmod \pi^{2 e-\nu+1}\right)$, we see that $L^{\prime} \cong K^{\prime}$ by the lemma and so $L \cong K$ by Theorem 2.1 .

4. In this section we shall make use of the Hasse invariant $S(\mathcal{L})$ $=\sum_{\lambda \leq \mu}\left(a_{\lambda}, a_{\mu}\right)$ of a (diagonal) quadratic form $\mathcal{L}=\sum a_{\lambda} x_{\lambda}^{2}$; this symbol is fully discussed by Witt in [10]. An easy computation [9] shows that if $\epsilon$ is a unit and if $\alpha \in 0$, then

$$
\begin{gathered}
(1+4 \alpha, \epsilon)=1, \\
(1+4 \alpha, \epsilon \pi)=1 \Leftrightarrow 1+4 \alpha=a^{2}, \quad a \in F .
\end{gathered}
$$

Durfee [2] has shown that two lattices $L$ and $K$ are equivalent if they are congruent modulo $4 \pi \mathfrak{a}\left(\xi_{n}\right)$ where $\xi_{n}$ is the last basis element in any canonical representation of $L$. The next theorem is designed to improve the degree of approximation but it will be necessary to make the additional assumption that $L$ and $K$ are in the same metric space $V$.

TheOREM 4.1. Let $L=\sum_{0} \xi_{\lambda}$ and $K=\sum_{0} \zeta_{\lambda}$ be two lattices in the same space $V$, both of the same type and such that $\mathfrak{a}(K)=\mathfrak{a}(L) \subseteq(2) \mathfrak{o}$. If $\left(\zeta_{\lambda}\right)$ is canonical and has the properties (i) $\left(\zeta_{1}^{2}\right) \mathcal{D}=N(K)$, (ii) $\xi_{\lambda} \xi_{\mu}$ $=\zeta_{\lambda} \zeta_{\mu}+\left\{\mathfrak{a}\left(\zeta_{\lambda}\right) \cdot \mathfrak{a}\left(\zeta_{\mu}\right)\right\}$, then $L \cong K$. 
Proof. First let us prove the following contention: $L \cong K$ if $\left(\xi_{\lambda}\right)$ is also canonical.

Proof OF Contention. We have $\xi_{\lambda} \xi_{\mu}=\zeta_{\lambda} \zeta_{\mu}$ if $\lambda \neq \mu ; \xi_{\lambda}^{2}=\zeta_{\lambda}^{2}+\left\{a\left(\zeta_{\lambda}\right)^{2}\right\}$. Put $\zeta_{\lambda}^{2}=a_{\lambda}$. There is no loss of generality in assuming that $a(K)$ $=a(L)=(2)$ o.

CASE 1. $\xi_{1}$ and $\zeta_{1}$ proper. Then $\left(\xi_{1}^{2}\right) \mathfrak{D}=\mathfrak{a}\left(\xi_{1}\right)=(2) \mathfrak{b}=\mathfrak{a}\left(\zeta_{1}\right)=\left(a_{1}\right) \mathfrak{D}$. Define $f=0$ or $f=1$, the choice being such that $f+e$ is odd; define $\xi_{0}^{2}=\pi^{-f}$ and put $L^{\prime}=0 \xi_{0} \oplus L, K^{\prime}=0 \xi_{0} \oplus K$; by Theorem 2.1 it suffices to prove that $L^{\prime} \cong K^{\prime}$.

Suppose that we have constructed a new canonical basis

$$
X_{0}, X_{1}, \Xi_{2}, \cdots, \Xi_{i-1}, \xi_{i}, \cdots, \xi_{n}, \quad 2 \leqq i \leqq n,
$$

for $L^{\prime}$ with the property

$$
\Xi_{\lambda}^{2}=a_{\lambda}, \quad 1<\lambda<i .
$$

We now show how to construct $\Xi_{i}$, that is another basis $X_{0}, X_{1}$, $\boldsymbol{\Xi}_{2}, \cdots, \boldsymbol{\Xi}_{i-1}, \boldsymbol{\Xi}_{i}, \xi_{i+1}, \cdots, \xi_{n}$ in which the property $(4.4)$ is valid for all $1<\lambda \leqq i$ : the vectors $X_{0}, X_{1}$ will be variables in this construction.

By varying $X_{0}, X_{1}$, and $\xi_{i}$ alone, suppose that we obtain another canonical basis (which we continue to write in the form (4.3)) such that $\xi_{i}^{2}=a_{i}+\epsilon \pi^{\Delta}$. Then $\Delta$ is unbounded. For if not, we can suppose that $\Delta$ is maximal; then $\Delta \geqq 2$ ord $\mathfrak{a}\left(\xi_{i}\right)$. (a) Let $\Delta+e$ be even. If we define $\alpha$ so that $\alpha^{2} X_{1}^{2} \equiv \epsilon \pi^{\Delta}\left(\bmod \pi^{\Delta+1}\right)$, then $\left|\alpha^{2}\right|=\left|\pi^{\Delta} / 2\right| \leqq 1$ and $\left|\alpha \mathfrak{a}\left(X_{1}\right) / \mathfrak{a}\left(\xi_{i}\right)\right|^{2} \leqq|2|$; hence (3.2) is satisfied and we can apply op $\left(\xi_{i} \rightarrow \xi_{i}+\alpha X_{1}\right)$ to obtain a greater $\Delta$. Hence (b) $\Delta+e$ must be odd; then $\Delta+f$ is even and in virtue of the perfectness of the residue class field we can define $\alpha$ so that $\alpha^{2} X_{0}^{2} \equiv \epsilon \pi^{\Delta}\left(\bmod \pi^{\Delta+1}\right)$; then $\left|\alpha^{2}\right|=\left|\pi^{\Delta+f}\right|$ $\leqq 1$ and $\left|\alpha \mathfrak{a}\left(X_{0}\right) / \mathfrak{a}\left(\xi_{i}\right)\right|^{2}=\left|\pi^{\Delta-f} / \mathfrak{a}\left(\xi_{i}\right)^{2}\right|$ which is integral when $f=0$; if $f=1,\left|\pi^{\Delta}\right| \neq\left|a\left(\xi_{i}\right)\right|^{2}$ since $\Delta+e$ odd implies $\Delta$ odd; hence

$$
\pi^{\Delta-f} / \mathfrak{a}\left(\xi_{i}\right)^{2}
$$

is still integral; (3.2) is therefore satisfied for general $f$ and we can apply op $\left(\xi_{i} \rightarrow \xi_{i}+\alpha X_{0}\right)$ to reduce $\left|\pi^{\Delta}\right|$. Hence $\Delta$ is unbounded.

By choosing a $\xi_{i}$ with a sufficiently high $\Delta$, e.g. $\Delta \geqq$ ord $\left(4 \pi \mathfrak{a}\left(\xi_{i}\right)\right)$, we can construct a $\Xi_{i}$ such that

$$
\Xi_{i}^{2}=a_{i}
$$

in virtue of Lemmas 3.1 and 3.2. This is the $\Xi_{i}$ required by the construction.

Proceeding in this way we arrive at a basis $X_{0}, X_{1}, \Xi_{2}, \cdots, \Xi_{n}$ for 
$L^{\prime}$ such that $\sum_{2}^{n} 0 \Xi_{\lambda} \cong \sum_{2}^{n} 0 \zeta_{\lambda}$. Then $0 X_{0} \oplus \nabla X_{1}$ and $0 \xi_{0} \oplus 0 \zeta_{1}$ are fractionally equivalent by Witt's theorem. Now $X_{0}^{2}$ must represent $\pi^{-f}(\bmod 2)$ and in virtue of (3.2) and (3.8) we can therefore apply op $\left(X_{0} \rightarrow X_{0}+\alpha X_{1}\right)$ and op $\left(X_{0} \rightarrow X_{0}+\alpha X_{0}\right)$ in succession to reduce $\mathrm{o} X_{0} \oplus \mathrm{o} X_{1}$ to the form $X_{0}^{2}=\pi^{-f} \delta$ and $X_{1}^{2}=a_{1} \delta$ where $\delta \equiv 1(\bmod 4)$. Applying Hasse symbols to $\mathrm{o} X_{0} \oplus \mathrm{o} X_{1}$ and $\mathrm{o} \xi_{0} \oplus \mathrm{o} \zeta_{1}$ we get $\left(\pi^{-f} \delta,-1\right)$ $\cdot\left(a_{1} \delta, a_{1} \pi^{-f}\right)=\left(\pi^{-f},-1\right)\left(a_{1}, a_{1} \pi^{-f}\right)$. Hence $\left(\delta,-a_{1} \pi^{f}\right)=1$; but ord $a_{1} \pi^{f}$ $=e+f$ is odd, by choice of $f$; hence $\delta$ is a square by (4.2). This completes the proof of the first case.

CASE 2. $\xi_{1}$ and $\zeta_{1}$ improper. Since $N(L)=\left(\zeta_{1}^{2}\right) 0=\left(2 \pi^{\nu}\right)$ o where $0<\nu \leqq e$, we can suppose that $\left(\xi_{1}^{2}\right) D=N(L)$. Define $f=0$ or $f=1$, in such a way that $f+\nu+e$ is odd. Define $L^{\prime}$ and $K^{\prime}$ as in Case 1 . We must prove that $L^{\prime} \cong K^{\prime}$.

Suppose that we have constructed a canonical basis

$$
X_{0}, X_{1}, X_{2}, \Xi_{3}, \cdots, \Xi_{i-1}, \xi_{i}, \cdots, \xi_{n}, \quad 3 \leqq i \leqq n ;
$$

for $L^{\prime}$, having the following properties

$$
\begin{array}{r}
X_{1}^{2} \equiv \xi_{1}^{2}(\bmod 4 \pi), \quad \Xi_{\lambda}^{2}=a_{\lambda} \text { for } 2<\lambda<i, \\
N(L)=N\left(\mathrm{o} X_{1}+\mathrm{o} X_{2}+\mathrm{D} \Xi_{3}+\cdots+\mathrm{o} \xi_{n}\right) .
\end{array}
$$

We now give the construction for $\Xi_{i}$ : that is, we produce another basis $X_{0}, X_{1}, X_{2}, \Xi_{3}, \cdots, \Xi_{i-1}, \Xi_{i}, \xi_{i+1}, \cdots, \xi_{n}$ for $L^{\prime}$, having the properties (4.7) and (4.8) for all $2<\lambda \leqq i ; X_{0}, X_{1}, X_{2}$ will be variable vectors in this construction. Note that (4.7) and (4.8) together imply that $\left(X_{1}^{2}\right) \mathfrak{D}=N\left(\mathrm{o} X_{1}+\mathrm{o} X_{2}+\mathrm{o} \Xi_{3}+\cdots+\mathrm{o} \xi_{n}\right)$ and hence

$$
\left|X_{2}^{2}\right| \leqq\left|X_{1}^{2}\right| \text {. }
$$

By varying $X_{0}, X_{1}, X_{2}$ and $\xi_{i}$ alone, suppose that we obtain another canonical basis (which we continue to write in the form (4.6)) with $\xi_{i}^{2}=a_{i}+\epsilon \pi^{\Delta}$ and such that the new basis continues to satisfy equations (4.7) and (4.8). Then $\Delta$ is unbounded. For if not, we can choose a $\Delta$ that is maximal; then $\Delta \geqq 2$ ord $\left(\mathfrak{a}\left(\xi_{i}\right)\right)$. (a) Let $\Delta+e+\nu$ be even. In virtue of the perfectness of the residue class field we can define $\alpha$ so that $\alpha^{2} X_{1}^{2} \equiv \epsilon \pi^{\Delta}\left(\bmod \pi^{\Delta+1}\right)$; then $\left|\alpha^{2}\right|=\left|\pi^{\Delta} / 2 \pi^{\nu}\right| \leqq 1$; and

$$
\left|\alpha \mathfrak{a}\left(X_{1}\right) / \mathfrak{a}\left(\xi_{i}\right)\right|^{2} \leqq 1 ;
$$

hence (3.2) is satisfied and we can apply op $\left(\xi_{i} \rightarrow \xi_{i}+\alpha X_{1}\right)$; under this change of basis the new value of $X_{1}$ satisfies (4.7) in virtue of (3.6) and (3.7), (4.8) is obviously true, and $\xi_{i}+\alpha X_{1}$ has a greater $\Delta$, denying the maximality of $\Delta$. Hence (b) $\Delta+e+\nu$ must be odd and so 
$\Delta+f$ is even. As in case $1(\mathrm{~b})$ we see that this is also impossible. Hence $\Delta$ is unbounded.

We now define $\boldsymbol{\Xi}_{i}$ as in (4.5). Proceeding in this way we obtain a basis $X_{0}, X_{1}, X_{2}, \Xi_{3}, \cdots, \Xi_{n}$ for $L^{\prime}$, which satisfies (4.7) and (4.8) and is such that $\sum_{3}^{n} \mathrm{D} \Xi_{\lambda} \cong \sum_{3}^{n} \mathrm{o} \zeta_{\lambda}$. By Witt's theorem, $L^{\prime \prime}=\mathrm{o} X_{0}$ $\oplus\left(\mathrm{o} X_{1}+\mathrm{o} X_{2}\right)$ and $K^{\prime \prime}=\mathrm{D} \xi_{0} \oplus\left(\mathrm{D} \zeta_{1}+0 \zeta_{2}\right)$ are field equivalent; the rest of the proof is devoted to proving that they are in fact integrally equivalent.

Let $L^{\prime \prime}=\mathrm{o} X_{0} \oplus\left(\mathrm{o} X_{1}+\mathrm{o} X_{2}\right)$ be a canonical basis for $L^{\prime \prime}$ that satisfies (4.9) and write $X_{1}^{2}=a_{1}+\epsilon \pi^{\Delta}$. Then $\Delta$ is unbounded. For if not, we can choose a $\Delta$ that is maximal; then $\Delta \geqq 2 e$ by (4.7); and we can assume that $\left(X_{1}^{2}\right)_{D}=N(L)$. (a) Let $\Delta+e+\nu$ be even. If $\Delta<3 e+\nu$, by the perfectness of the residue class field and by (3.8) there is an $\alpha$ such that $(1+\alpha)^{2} X_{1}^{2} \equiv a_{1}\left(\bmod \pi^{\Delta+1}\right)$; applying op $\left(X_{1} \rightarrow X_{1}+\alpha X_{1}\right)$ with this $\alpha$ leads to a contradiction. By Lemma 3.2 we see that $\Delta \geqq 3 e+\nu$ is also impossible. Hence (b) $\Delta+e+\nu$ is odd and so $\Delta+f$ is even. This is again impossible as in case 1 (b). Hence $\Delta$ is unbounded and we can take $\Delta=\infty$ by Lemma 3.2. We fix this vector for which $X_{1}^{2}=a_{1}$. Now let $X_{2}^{2}=a_{2}+\epsilon \pi^{\Delta}$; if $\Delta$ is unbounded as $X_{0}$ and $X_{2}$ vary, we are through; if not, let $X_{2}$ be chosen so that $\Delta$ is maximal; if $\Delta<3 e-\nu$, then $\Delta \geqq e+\nu$ by (4.9), and so $\Delta+e+\nu$ must be odd by (3.9); hence $\Delta+f$ must be even; now $X_{0}^{2} \cong \pi^{-f}\left(1+\left\{2 \pi^{\nu+f}\right\}\right)$ and a determinantal consideration therefore shows that $\Delta \geqq 2 e+f$; applying op $\left(X_{2} \rightarrow X_{2}+\alpha X_{0}\right)$ with $\alpha^{2} X_{0}^{2} \equiv \epsilon \pi^{\Delta}\left(\bmod \pi^{\Delta+1}\right)$ shows that this is impossible. Hence $\Delta \geqq 3 e-\nu$.

We therefore have a basis $\mathrm{o} X_{0} \oplus\left(\mathfrak{o} X_{1}+\mathfrak{o} X_{2}\right)$ for $L^{\prime \prime}$ such that $X_{1}^{2}=a_{1}, X_{2}^{2}=a_{2}+\left\{\pi^{3 e-\nu}\right\}, X_{1} X_{2}=\pi^{e}$ and therefore $X_{0}^{2}=\pi^{-f} \delta$ where $\delta \equiv 1(\bmod 4)$. Applying Hasse symbols to $L^{\prime \prime}$ and $K^{\prime \prime}$ we get

$$
\left(\pi^{-f} \delta,-1\right)\left(a_{1}, a_{1} \pi^{-f} \delta\right)\left(a_{1} d \delta, d \pi^{-f}\right)=\left(\pi^{-f},-1\right)\left(a_{1}, a_{1} \pi^{-f}\right)\left(a_{1} d, d \pi^{-f}\right)
$$

where $d$ denotes the determinant of $0 \zeta_{1}+o \zeta_{2}$. Hence $(\delta, \pi)=1$ and so $\delta$ is a square by (4.2). Hence $L^{\prime \prime} \cong K^{\prime \prime}$ by Corollary 3.2. Q.E.D.

PROOF OF THEOREM. First we show how to construct a canonical basis $\left(\boldsymbol{\Xi}_{\lambda}\right)$ for $L$ from the given basis $\left(\xi_{\lambda}\right)$. Suppose that $\boldsymbol{\Xi}_{\lambda}=\xi_{\lambda}+\alpha_{\lambda} X_{\lambda}$ has already been defined for $0<\lambda<i$ where $1 \leqq i \leqq n$, and such that $\alpha_{\lambda} \in \mathfrak{a}\left(\zeta_{\lambda}\right), X_{\lambda} \in L, \zeta_{i-1} \zeta_{i}=0, L=\sum_{1}^{i-1} 0 \Xi_{\lambda}+\sum_{i}^{n} 0 \xi_{\lambda}$ where $0 \Xi_{1}+\cdots$ $+D \Xi_{i-1}$ is canonical with the same type as $0 \zeta_{1}+\cdots+D \zeta_{i-1}$. We now define $\boldsymbol{\Xi}_{\boldsymbol{i}}$.

CASE 1. $\zeta_{i}$ is proper. Consider the vector $Y=\xi_{i}+A_{1} \Xi_{1}+\ldots$ $+A_{i-1} \Xi_{i-1}$. An easy computation shows that $A_{j} \in \mathfrak{a}\left(\zeta_{i}\right)$ exist such that $Y \Xi_{\lambda}=0, \lambda<i$; the $Y$ corresponding to these $A_{j}$ is defined to be $\boldsymbol{\Xi}_{\boldsymbol{i}}$. 
CASE 2. $\zeta_{i}$ improper. Define $\boldsymbol{\Xi}_{i}$ and $\boldsymbol{\Xi}_{t+1}^{\prime}$ as in Case 1. Then $\boldsymbol{\Xi}_{i} \boldsymbol{\Xi}_{i+1}^{\prime}$ $=\zeta_{i} \zeta_{i+1}\left(1+\left\{\mathfrak{a}\left(\zeta_{i}\right)\right\}\right)$ and we put $\Xi_{i+1}=\Xi_{i+1}^{\prime}\left(\zeta_{i} \zeta_{i+1} / \Xi_{i} \Xi_{i+1}^{\prime}\right)$.

Proceeding in this manner we arrive at a canonical basis $\left(\Xi_{\lambda}\right)$ for $L$ such that $\boldsymbol{\Xi}_{\lambda}^{2}=\left(\xi_{\lambda}+\alpha_{\lambda} X_{\lambda}\right)^{2}=\zeta_{\lambda}^{2}+\left\{\mathfrak{a}\left(\zeta_{\lambda}\right)^{2}\right\}$. The conditions of the theorem are therefore satisfied by the canonical basis $\left(\Xi_{\lambda}\right)$ and so $L$ is isometric to $K$.

THEOREM 4.2. Let $L=L_{1} \oplus L_{2}=K_{1} \oplus K_{2}$ be two decompositions of the totally integral lattice $L$ such that $L_{1}$ and $K_{1}$ are of unit determinant and $\mathfrak{a}\left(L_{2}\right)=\mathfrak{a}\left(K_{2}\right) \subseteq(2) \mathfrak{o}$. If $L_{1} \cong K_{1}$, then $L_{2} \cong K_{2}$.

Proof. First we assume that $L_{1}$ and $K_{1}$ are proper. Write $L_{1}=0 \xi_{1}$ $\oplus \cdots \oplus \mathrm{o} \xi_{r-1} ; L_{2}=\mathrm{o} \xi_{r}+\cdots+\mathrm{o} \xi_{n} ; K_{1}=\mathrm{o} \eta_{1} \oplus \cdots \oplus \mathrm{o} \eta_{r-1} ; K_{2}=\mathrm{o} \eta_{r}$ $+\cdots+o \eta_{n} ;$ all these bases are canonical and such that $\left(\xi_{r}^{2}\right) \mathfrak{o}=N\left(L_{2}\right)$. Define

$$
\Xi_{\lambda}=\xi_{\lambda}-\left(\xi_{\lambda} \eta_{1} / \eta_{1}^{2}\right) \eta_{1}-\cdots-\left(\xi_{\lambda} \eta_{r-1} / \eta_{r-1}^{2}\right) \eta_{r-1}
$$

for $r \leqq \lambda \leqq n$. Then $\Xi_{\lambda}=\xi_{\lambda}+\left\{a\left(\xi_{\lambda}\right)\right\} X_{\lambda}$ where $X_{\lambda} \in L$; and $L=L_{1}$ $+\sum_{r}^{n} \mathrm{D} \Xi_{\lambda}$ since $\mathfrak{a}\left(\xi_{\lambda}\right) \subseteq(2) \mathrm{D}$; and $\sum_{r}^{n} \mathrm{D} \Xi_{\lambda} \subseteq K_{2}$ since $\Xi_{\lambda} \eta_{\mu}=0$ for $1 \leqq \mu \leqq r-1$; now in general the change of basis in a lattice is effected by a unimodular transformation and a determinantal consideration of the matrix connecting $\eta_{1}, \cdots, \eta_{n}$ and $\xi_{1}, \cdots, \xi_{r-1}, \Xi_{r}, \cdots, \Xi_{n}$ therefore shows that $\sum_{r}^{n} 0 \Xi_{\lambda}=K_{2}$. But $L_{2}$ and $\sum_{r}^{n} 0 \Xi_{\lambda}$ are field equivalent by Witt's theorem; and $\Xi_{\lambda} \Xi_{\mu}=\xi_{\lambda} \xi_{\mu}+\left\{\mathfrak{a}\left(\xi_{\lambda}\right) \mathfrak{a}\left(\xi_{\mu}\right)\right\}$; hence $L_{2} \cong K_{2}$ by Theorem 4.1 .

If $L_{1}$ and $K_{1}$ are improper, define $\xi_{0}^{2}=1$ and put $L^{\prime}=0 \xi_{0} \oplus L$ $=L_{1}^{\prime} \oplus L_{2}=K_{1}^{\prime} \oplus K_{2}$. Then $L_{1}^{\prime}$ and $K_{1}^{\prime}$ are proper and so $L_{2} \cong K_{2}$.

5. We shall call a vector $X \in V$ isotropic if $X^{2}=0$; a binary lattice $L$ will be called totally isotropic if it has a basis $L=\mathfrak{o} X+\mathrm{o} Y$ for which $X^{2}=0=Y^{2}$.

LEMMA 5.1. Let $L$ be any (not necessarily integral) lattice in $V$ and $\xi_{1}, \xi$ isotropic vectors in $L$ such that $L=\left(0 \xi_{1}+o \xi\right) \oplus L_{1}$ and $\left|\xi_{1} \xi\right|=1$. If $\eta$ is an isotropic vector in $L$ such that $|\xi \eta|=1$ and $\mathfrak{a}(\eta)=0$, then $L=(0 \eta+0 \xi) \oplus L_{1}^{\prime}$ and $L_{1}^{\prime}$ is isometric to $L_{1}$.

Proof. We have $\eta=\alpha \xi_{1}+\beta \xi+X$ with $X \in L_{1}$; in virtue of the assumption $\left|\xi_{\eta}\right|=1, \alpha$ must be a unit. Hence we can write $L=\left(\mathrm{o} \xi_{1}\right.$ $+0 \xi) \oplus \sum 0 \xi_{\lambda}=(0 \eta+0 \xi)+\sum 0 \xi_{\lambda}=(0 \eta+0 \xi) \oplus \sum 0 \Xi_{\lambda}$ where $\Xi_{\lambda}=\left(\xi_{\lambda}\right.$ $\left.-\left(\eta \xi_{\lambda} / \eta \xi\right) \xi\right)$, since $\eta \xi_{\lambda} / \eta \xi \in \mathbb{0}$. Put $L_{1}^{\prime}=\sum 0 \Xi_{\lambda} ;$ this proves the first part of the lemma.

Now $\boldsymbol{\Xi}_{\lambda} \boldsymbol{\Xi}_{\mu}=\left(\xi_{\lambda}-\left(\eta \xi_{\lambda} / \eta \xi\right) \xi\right) .\left(\xi_{\mu}-\left(\eta \xi_{\mu} / \eta \xi\right) \xi\right)=\xi_{\lambda} \xi_{\mu}$ since $\xi$ is isotropic and orthogonal to both $\xi_{\lambda}$ and $\xi_{\mu}$. Hence $L_{1}^{\prime} \cong L_{1}$. Q.E.D. 
If $L$ is a totally integral lattice of unit determinant and of dimension $\geqq 3$, there is a decomposition

$$
L=\sum N_{\lambda} \oplus L_{1} \oplus L_{2}
$$

where the $N_{\lambda}$ are totally isotropic binary lattices of unit determinant and where $L_{1}, L_{2}$ satisfy the following conditions: if $L$ is proper, $L_{2} \cong[1] \oplus[1]$ or $L_{2} \cong[1]$, while if $L$ is improper,

$$
L_{2} \cong\left(\begin{array}{cc}
{\left[\pi^{\nu}\right]} & 1 \\
1 & \left\{\pi^{\nu}\right\}
\end{array}\right), \quad \quad \nu>0 ;
$$

and

$$
L_{1} \cong\left(\begin{array}{cc}
{\left[\pi^{\mu}\right]} & 1 \\
1 & \{2\}
\end{array}\right), \quad \text { with } \mu>0
$$

for $L$ proper, and with $\mu>\nu>0$ for $L$ improper. Note that in this decomposition $L_{1}$ is totally isotropic if $\mu>e$.

In the decomposition (5.1) the term $\sum N_{\lambda}$ is omitted when $\operatorname{dim} L$ is either 3 or 4 , in other words we then have $L=L_{1} \oplus L_{2}$. We extend this decomposition to lattices of lower dimension by writing

$$
L \cong L_{2} \quad \text { if } \operatorname{dim} L \leqq 2 ;
$$

the value $\mu=\infty$ is associated with such a lattice.

The proof that a decomposition (5.1) does in fact exist is a simple application of the operations op $\left(\xi_{i} \rightarrow \xi_{i}+\alpha \xi_{j}\right)$ of $\S 3$ and is left to the reader. Compare the result in [9].

THEOREM 5.2. Let $L_{1} \oplus\left(\mathrm{o} \xi_{j}+\mathrm{o} \xi_{j+1}\right) \oplus L_{2}=L=K_{1} \oplus\left(\mathrm{o} \eta_{j}+\mathrm{o} \eta_{j+1}\right) \oplus K_{2}$ be two decompositions of the lattice $L$ such that $\mathrm{o} \xi_{j}+\mathrm{o} \xi_{j+1}$ and $\mathrm{o} \eta_{j}+\mathrm{o} \eta_{j+1}$ are totally isotropic and isometric. Then $L_{1} \oplus L_{2}$ and $K_{1} \oplus K_{2}$ are isometric.

Proof. There is no loss of generality in taking $a\left(\xi_{j}\right)=\mathfrak{D}=\mathfrak{a}\left(\eta_{j}\right)$. In virtue of the canonical form decomposition theorem and of (5.1) we can write

$$
L=\sum_{1}^{j-1} 0 \xi_{\lambda} \oplus\left(0 \xi_{j}+0 \xi_{j+1}\right) \oplus \sum_{j+2}^{k} 0 \xi_{\lambda} \oplus \sum_{k+1}^{n} 0 \xi_{\lambda}
$$

where (i) $\left|\mathfrak{a}\left(\xi_{\lambda}\right)\right|>1$ for $1 \leqq \lambda \leqq j-1,\left|\mathfrak{a}\left(\xi_{\lambda}\right)\right|=1$ for $j \leqq \lambda \leqq k$, and $\left|\mathfrak{a}\left(\xi_{\lambda}\right)\right|<1$ for $k+1 \leqq \lambda \leqq n$; (ii) $\xi_{\lambda}$ is canonical for $1 \leqq \lambda \leqq j-1$ and for $k+1 \leqq \lambda \leqq n$; (iii) $\sum_{j+2}^{k} \circ \xi_{\lambda}$ is expressed in the form (5.1). Since the lattice $\sum_{j+2}^{k} D \xi_{\lambda}$ has the form (5.1), we can define integers $\nu$ and $\mu$ corresponding to the basis $\left(\xi_{j+2}, \cdots, \xi_{k}\right)$. Of course $\nu$ and $\mu$ will vary 
as we alter the basis $\left(\xi_{1}, \cdots, \xi_{j-1}, \xi_{j+2}, \cdots, \xi_{n}\right)$ : of all such bases for $L_{1} \oplus L_{2}$ which have the properties (i) to (iii), choose one in which first $\nu$ and then $\mu$ is maximal ( $\nu$ will be zero when $a\left(\xi_{k}\right)=0$ ). Note that $\xi_{j}$ and $\xi_{j+1}$ are unaltered in this choice of basis. We shall refer to this new basis as $\left(\xi_{\lambda}\right)$. We can assume that both $\xi_{\lambda}$ and $\xi_{\lambda+1}$ are isotropic vectors whenever $0 \xi_{\lambda}+0 \xi_{\lambda+1}$ is totally isotropic.

Contention: there is an isotropic vector $\xi_{i}, j \leqq i \leqq k$, such that (a) either $\left|\xi_{i} \eta_{j}\right|=1$ or $\left|\xi_{i} \eta_{j+1}\right|=1$; (b) if $\xi_{i} \xi_{i+\delta} \neq 00$, then $\xi_{i+\delta}$ is also isotropic.

Proof of Contention. Write

$$
\begin{aligned}
\eta_{j} & =a_{1 j} \xi_{1}+\cdots+a_{j j} \xi_{j}+\cdots+a_{k j} \xi_{k}+\cdots+a_{n j} \xi_{n} \\
& =X_{i}+a_{j i} \xi_{j}+\cdots+a_{k j} \xi_{k}+Y_{j}
\end{aligned}
$$

where $a_{\lambda j} \in$ o. Similarly

$$
\eta_{j+1}=X_{j+1}+a_{j j+1} \xi_{j}+\cdots+a_{k j+1} \xi_{k}+Y_{j+1} .
$$

Let us consider any $\lambda, 1 \leqq \lambda \leqq j-1$. If $\xi_{\lambda}$ is proper, then $\left|\eta_{j} \xi_{\lambda}\right| \leqq 1$ implies that $\left|a_{\lambda j} \xi_{\lambda}^{2}\right| \leqq 1$ and so $\left|a_{\lambda j}\right|<1$; similarly $\left|a_{\lambda j+1}\right|<1$; hence $\left|a_{\lambda j}^{2} \xi_{\lambda}^{2}\right|<1,\left|a_{\lambda j+1}^{2} \xi_{\lambda}^{2}\right|<1$ and $\left|a_{\lambda j} a_{\lambda j+1} \xi_{\lambda}^{2}\right|<1$. If $\xi_{\lambda}$ is improper $\left(\xi_{\lambda} \xi_{\lambda+1}\right.$ $\neq 0$, say), then $\left|\eta_{j} \xi_{\lambda}\right| \leqq 1$ implies that $\left|a_{\lambda j} \xi_{\lambda}^{2}+a_{\lambda+1} \xi_{\lambda} \xi_{\lambda+1}\right| \leqq 1$ and so $\left|a_{\lambda+1 j}\right|<1$ and by symmetry $\left|a_{\lambda j}\right|<1$; then

$$
\left|\eta_{j}\left(a_{\lambda j} \pi^{-1} \xi_{\lambda}+a_{\lambda+1} \pi^{-1} \xi_{\lambda+1}\right)\right| \leqq 1
$$

implies that $\left(a_{\lambda j} \xi_{\lambda}+a_{\lambda+1} \xi_{\lambda+1}\right)^{2} \in(\pi) 0$; similarly

$$
\left(a_{\lambda j+1} \xi_{\lambda}+a_{\lambda+1 j+1} \xi_{\lambda+1}\right)^{2} \in(\pi) 0
$$

and

$$
\left(a_{\lambda j} \xi_{\lambda}+a_{\lambda+1 j} \xi_{\lambda+1}\right)\left(a_{\lambda j+1} \xi_{\lambda}+a_{\lambda+1 j+1} \xi_{\lambda+1}\right) \in(\pi) \mathbf{0} .
$$

Hence we have

$$
X_{j}^{2} \in(\pi) \mathrm{o}, \quad X_{j} X_{j+1} \in(\pi) \mathrm{o}, \quad X_{j+1}^{2} \in(\pi) \text { o. }
$$

It is easy to see that

$$
Y_{j}^{2} \in(\pi) \mathrm{o}, \quad Y_{j} Y_{j+1} \in(\pi) \mathrm{o}, \quad Y_{j+1}^{2} \in(\pi) \mathrm{o} .
$$

Now let us consider the following cases.

CASE $1 . k-(j+1)=0$. Then $\eta_{j} \eta_{j+1}$ is a unit, but $\left|X_{j} X_{j+1}\right|<1$ and $\left|Y_{j} Y_{j+1}\right|<1$; hence $a_{j j}$ (say) must be a unit and $\xi_{i}=\xi_{j+1}$ is the vector required.

CASE 2. $k-(j+1)>0$ is odd. An easy computation shows that $a_{k j} \in(\pi)$ o. But $\left|\eta_{j} \eta_{j+1}\right|=1$ and so at least one $a_{\lambda j}$ must be a unit, 
$j \leqq \lambda \leqq k-1$. If $\mu>e$ or if $k=j+2$, we are through. So assume that $k>j+2$ and $\mu \leqq e$. Let us suppose (if possible) that $\left|a_{k-2 j}\right|=1$ but $\left|a_{\lambda j}\right|<1$ for $j \leqq \lambda \leqq k-3$. Put $\Xi_{k-2}=a_{1 j} \xi_{1}+\cdots+a_{j-1} \xi_{j-1}+a_{k-2 j} \xi_{k-2}$ $+\cdots+a_{n j} \xi_{n}$ and $\Xi_{k-1}=\xi_{k-1}$. Then $\Xi_{k-2}^{2} \in(2 \pi) 0$ since $\eta_{j}^{2}=0$. Define $\Xi_{\lambda}=\xi_{\lambda}+A_{\lambda} \Xi_{k-2}+B_{\lambda} \Xi_{k-1}$ for $1 \leqq \lambda \leqq j-1, k \leqq \lambda \leqq n$, where $A_{\lambda}$ and $B_{\lambda}$ are the integral solutions to

$$
\begin{aligned}
& \xi_{\lambda} \Xi_{k-2}+A_{\lambda} \Xi_{k-2}^{2}+B_{\lambda} \Xi_{k-2} \Xi_{k-1}=0, \\
& \xi_{\lambda} \Xi_{k-1}+A_{\lambda} \Xi_{k-2} \Xi_{k-1}+B_{\lambda} \Xi_{k-1}^{2}=0 .
\end{aligned}
$$

Then $\sum_{1}^{j-1} \mathrm{D} \xi_{\lambda} \oplus \sum_{k-2}^{n} \mathrm{D} \xi_{\lambda}=\sum_{1}^{j-1} \mathrm{D} \Xi_{\lambda}+\left(0 \Xi_{k-2}+0 \Xi_{k-1}\right)+\sum_{k}^{n} 0 \Xi_{\lambda}$; if we put $\sum_{1}^{j-1} 0 \Xi_{\lambda}+\sum_{k}^{n} D \Xi_{\lambda}$ into canonical form and consider the resulting form for $L$, we see that $\Xi_{k-2}^{2} \in(2 \pi)_{0}$ denies the maximality of $\mu$. Hence $\left|a_{k-2 j}\right|<1$ if $\left|a_{\lambda j}\right|<1$ for all $j \leqq \lambda \leqq k-3$; and similarly $\left|a_{k-2 j+1}\right|<1$ if $\left|a_{\lambda j+1}\right|<1$ for $j \leqq \lambda \leqq k-3$. But these inequalities cannot be satisfied simultaneously since $\left|\eta_{j} \eta_{j+1}\right|=1$; hence at least one $a_{\lambda j}$ or one $a_{\lambda j+1}, j \leqq \lambda \leqq k-3$, must be a unit and this completes the proof of the second case.

CASE 3. $k-(j+1)>0$ is even.

(a) $0 \xi_{k-1}+0 \xi_{k}$ proper. Since $\eta_{j}^{2}=0=\eta_{j+1}^{2}$, we must have $a_{k-1}^{2} \xi_{k-1}^{2}$ $+a_{k j}^{2} \xi_{k}^{2} \equiv 0(\bmod \pi)$ and $a_{k-1 j+1}^{2} \xi_{k-1}^{2}+a_{k j+1}^{2} \xi_{k}^{2} \equiv 0(\bmod \pi)$ from which it follows that

$$
a_{k-1 j} a_{k-1 j+1} \xi_{k-1}^{2}+a_{k j} a_{k j+1} \xi_{k}^{2} \equiv 0(\bmod \pi) .
$$

Hence at least one $a_{\lambda j}, j \leqq \lambda \leqq k-2$, must be a unit. If $k=j+3$ or if $\mu>e$ we are through. If $\mu \leqq e$ and $k>j+3$ we can employ the argument of Case 2 to show that at least one $a_{\lambda j}$ or one $a_{\lambda j+1}, j \leqq \lambda \leqq k-4$, must be a unit.

(b) $\mathfrak{D} \xi_{k-1}+\mathfrak{D} \xi_{k}$ improper. If $\nu>e$ we can assume that $\xi_{k-1}^{2}=0=\xi_{k}^{2}$ by (3.10) and Lemma 3.2; hence that $\mu>e$; the result is an immediate consequence of the equation $\left|\eta_{j} \eta_{j+1}\right|=1$. Now let $\nu=e$ (and so $\mu>e$ again): suppose, if possible, that $\left|a_{k-1 j}\right|=1$ but $\left|a_{\lambda j}\right|<1$ for all $i \leqq \lambda \leqq k-2$. Define

$$
\Xi_{k-1}=a_{1 j} \xi_{1}+\cdots+a_{j-1} \xi_{j-1}+a_{k-1} \xi_{k-1}+\cdots+a_{n j} \xi_{n} .
$$

Then $\Xi_{k-1}^{2} \in(2 \pi)$ o. Write $\Xi_{k}=\xi_{k}$ and define $\Xi_{\lambda}$ as in (5.7) for $1 \leqq \lambda$ $\leqq j-1, k+1 \leqq \lambda \leqq n$. Then $\sum_{1}^{j-1} \mathrm{o} \xi_{\lambda} \oplus \sum_{k-1}^{n} 0 \xi_{\lambda}=\sum_{1}^{j-1} \mathrm{D} \Xi_{\lambda}+\left(0 \Xi_{k-1}\right.$ $\left.+0 \Xi_{k}\right)+\sum_{k+1}^{n} D \Xi_{\lambda}$ and this denies the maximality of $\nu$. Hence there is at least one $a_{\lambda j}$ or one $a_{\lambda j+1}, j \leqq \lambda \leqq k-2$, such that $\left|a_{\lambda j}\right|=1$. If $k=j+3$ we are through; if $k>j+3,0 \xi_{k-3}+0 \xi_{k-2}$ is totally isotropic, and so we are through. Now let $\nu<e$; then we can assume that $\left|\xi_{k}^{2}\right|<\left|\pi^{\nu}\right|$ by (3.9). Using this fact in an argument similar to the one 
just given, we see that $\left|a_{k-1 j}\right|<1$ and $\left|a_{k-1 j+1}\right|<1$ must always hold, no matter what the values of $a_{\lambda j}$ and $a_{\lambda j+1}, j \leqq \lambda \leqq k-2$, may be. If $k=j+3$ or if $\mu>e$, we are through. So let $k>j+3$ and $\mu \leqq e$. Suppose, if possible, that $\left|a_{k-3 j}\right|=1$ but $\left|a_{\lambda j}\right|<1$ for all $j \leqq \lambda \leqq k-4$. Define

$$
\Xi_{k-3}^{\prime}=\sum_{1}^{j-1} a_{\lambda j} \xi_{\lambda}+a_{k-3 j} \xi_{k-3}+a_{k-2 j} \xi_{k-2}+\sum_{k+1}^{n} a_{\lambda j} \xi_{\lambda}
$$

and $\boldsymbol{\Xi}_{k-3}=\Xi_{k-3}^{\prime}+a_{k-1} \xi_{k-1}+a_{k j} \xi_{k}$. Then, as in Case 2, we have

$$
\begin{aligned}
\sum_{1}^{1-1} 0 \xi_{\lambda} \oplus\left(0 \xi_{k-3}\right. & \left.+0 \xi_{k-2}\right) \oplus\left(0 \xi_{k-1}+0 \xi_{k}\right) \oplus \sum_{k+1}^{n} 0 \xi_{\lambda} \\
& =\sum_{1}^{j-1} 0 \Xi_{\lambda}+\left(\left(0 \Xi_{k-3}^{\prime}+0 \xi_{k-2}\right) \oplus\left(0 \xi_{k-1}+0 \xi_{k}\right)\right)+\sum_{k+1}^{n} 0 \Xi_{\lambda} \\
& =\sum_{1}^{j-1} 0 \Xi_{\lambda}+\left(\left(0 \Xi_{k-3}+0 \xi_{k-2}\right) \oplus\left(0 \Xi_{k-1}+0 \Xi_{k}\right)\right)+\sum_{k+1}^{n} 0 \Xi_{\lambda}
\end{aligned}
$$

and by considering the norm of the middle blocks it is easy to see that we have $\left|\Xi_{k-1}^{2}\right| \leqq\left|\pi^{\nu}\right|$ and $\left|\Xi_{k}^{2}\right| \leqq\left|\pi^{\nu}\right|$; but we also have $\left|\Xi_{k-3}^{2}\right|<|2|$ $\leqq\left|\pi^{\mu}\right|$; this denies the maximality of $\mu$. Hence there is at least one $a_{\lambda j}$ or one $a_{\lambda j+1}, j \leqq \lambda \leqq k-4$, which is a unit. This completes the proof of the contention.

There is therefore a pair of isotropic vectors $\xi_{i}, \xi_{i+\delta}$, such that $\left|\eta_{j} \xi_{i}\right|=1$ (say), $\mathfrak{a}\left(\xi_{i}\right)=\mathrm{D}$ and $\xi_{i} \xi_{i+\delta}=1$ where $\delta= \pm 1$. Applying Lemma 5.1 twice we get $L=\left(0 \xi_{i}+o \xi_{i+\delta}\right) \oplus L_{3}=\left(0 \xi_{i}+o \eta_{j}\right) \oplus L_{4}=\left(o \eta_{j}\right.$ $\left.+o \eta_{j+1}\right) \oplus L_{5}$ with $L_{3} \cong L_{4} \cong L_{5}$. If $i=j$ or if $i=j+1$, we are through. If not, $L_{3}=\left(\mathrm{o} \xi_{j}+\mathrm{o} \xi_{j+1}\right) \oplus L_{3}^{\prime}$ and since $L_{3} \cong L_{5}$, we have $L_{5}=\left(\mathrm{o} X_{1}\right.$ $\left.+\mathrm{o} X_{2}\right) \oplus L_{5}^{\prime}$ with $\left(\mathrm{o} X_{1}+\mathrm{o} X_{2}\right) \cong\left(\mathrm{o} \xi_{j}+\mathrm{o} \xi_{j+1}\right)$ and $L_{3}^{\prime} \cong L_{6}^{\prime}$. Then $\left(\mathrm{o} \xi_{i}+\mathrm{o} \xi_{i+\delta}\right) \oplus L_{3}^{\prime} \cong\left(\mathrm{o} X_{1}+\mathrm{o} X_{2}\right) \oplus L_{6}^{\prime}$ : that is to say, $L_{1} \oplus L_{2} \cong K_{1} \oplus K_{2}$. Q.E.D.

This thorem establishes the second result announced in the introduction. For let $L=L^{\prime} \oplus L^{*}$ and $K=K^{\prime} \oplus K^{*}$ be isometric lattices with $L^{\prime} \cong \sum N_{\lambda} \cong K^{\prime}$ where $N_{\lambda}$ is of the form

$$
\left(\begin{array}{cc}
0 & \pi^{\nu(\lambda)} \\
\pi^{\nu(\lambda)} & 0
\end{array}\right) \text {. }
$$

If $\operatorname{dim} L^{\prime}=2=\operatorname{dim} K^{\prime}$, then $L^{*} \cong K^{*}$ by Theorem $5.2 ;$ if $\operatorname{dim} L^{\prime}$ $=\operatorname{dim} K^{\prime}>2$, we apply this result to cancel off two-dimensional totally isotropic lattices, one at a time, until we obtain $L^{*} \cong K^{*}$.

\section{REFERENCES}

1. E. Artin, Algebraic numbers and algebraic functions, Princeton, 1950. 
2. W. H. Durfee, Congruence of quadratic forms over valuation rings, Duke Math. J vol. 11 (1944) pp. 687-697.

3. M. Eichler, Quadratische Formen und orthogonale Gruppen, Berlin, 1952.

4. - Note zur Theorie der Kristallgitter, Math. Ann. vol. 125 (1952) pp. 5155.

5. B. W. Jones, An extension of a theorem of Witt, Bull. Amer. Math. Soc. vol. 48 (1942) pp. 133-142.

6. B. W. Jones and W. H. Durfee, A theorem on quadratic forms over the ring of 2-adic integers, Bull. Amer. Math. Soc. vol. 55 (1949) pp. 758-762.

7. B. W. Jones, The arithmetic theory of quadratic forms, Carus Monographs, no. $10,1950$.

8. M. Kneser, Zur Theorie der Kristallgitter, Math. Ann. vol. 127 (1954) pp. 105106.

9. O. T. O'Meara, Quadratic forms over local fields, Amer. J. Math. vol. 77 (1955) pp. 87-116.

10. E. Witt, Theorie der quadratischen Formen in beliebigen Körpern, J. Reine Angew. Math. vol. 176 (1937) pp. 31-44.

University of Otago, New Zealand 